\title{
DEPRESSION, LOWER URINARY TRACT SYMPTOMS AND QUALITY OF LIFE IN WOMEN WITH MULTIPLE SCLEROSIS: A DESCRIPTIVE AND CORRELATIONAL STUDY
}

\author{
Bruna Maciel Catarino', Alessandro Finkelsztejn², \\ Magda da Silva Aranchipe ${ }^{3}$, José Geraldo Lopes Ramos ${ }^{3}$, \\ Luciano Palmeiro Rodrigues ${ }^{1,4}$, Luciana Laureano Paiva ${ }^{1,4}$
}

\begin{abstract}
Introduction: Among the most ordinary clinical manifestations of Multiple Sclerosis (MS) are depression and the presence of Lower Urinary Tract Symptoms (LUTS). Both can compromise a person's quality of life. The objective of this research was to identify the major urinary symptoms and correlate them with quality of life and with depressive symptoms in women with MS.
\end{abstract}

Methods: This was an observatory, descriptive and correlational study, with nonprobabilistic sampling by convenience. This research included women over 18 years old who displayed LUT symptoms and who had been diagnosed with Relapsing-Remitting MS. Assessment consisted of an anamnesis card, the Kurtzke Expanded Disability Status Scale (EDSS), the Incontinence Impact Questionnaire-7 (IIQ-7-BR), the Urogenital Distress Inventory-6 (UDI-6-BR), the Beck Depression Inventory-2 (BDI-II) and the Multiple Sclerosis Quality of Life Questionnaire - Portuguese version (MSQOL-54).

Results: 41 women participated in the study, with average age of $50.1( \pm 9.45)$ and average of 4.11 in the EDSS. The most common urinary symptom was urinary urgency $(78 \%)$. There was no correlation between the severity of the urinary symptom and quality of life. Moderate and significant negative correlation $(r=-0.561 p<0.001)$ was found between depression and the physical component of quality of life and strong negative correlation $(r=-0.729 p<0.001)$ was found between depression and the mental component.

Conclusions: The most prevalent urinary symptom was urinary urgency. A strong correlation was found between symptoms of depression and quality of life and there was no correlation between urinary symptoms and quality of life.

Keywords: Multiple sclerosis; urinary incontinence; quality of life; depression

Multiple Sclerosis (MS) is a chronic, progressive, autoimmune, highly disabling disease ${ }^{1}$. It causes central nervous system (CNS) demyelination, in which the oligodendrocytes and myelin sheath are the main targets of inflammatory processes, causing motor, cognitive, sensory, and urogenital damage, interfering with the autonomy and functioning of patients ${ }^{1}$.

MS affects individuals between the ages of 20 and 40 and is more common in women, at a 2-by-1 ratio, which tends to increase and reach 3-by-12. In Brazil, the prevalence of MS varies greatly according to geographical distribution. The city with the highest prevalence rate is Santa Maria, state of Rio Grande do Sul, with 27.2 cases per 100,000 inhabitants $^{3}$. MS can be classified into four subtypes, depending on the clinical course of the disease ${ }^{2}$. The most frequent is Relapsing-Remitting MS (or remission), in which episodes of exacerbation of symptoms are interspersed with intervals or remissions, presenting a slow progression. The most common clinical manifestations are: fatigue, loss of strength, diplopia, sensitivity and coordination alterations, emotional changes and urogenital dysfunctions ${ }^{2}$.

\author{
Clin Biomed Res. 2019;39(3):193-199 \\ 1 Escola de Educação Física, Fisioterapia \\ e Dança (Esefid), Universidade Federal \\ do Rio Grande do Sul (UFRGS). \\ Porto Alegre, RS, Brasil. \\ 2 Serviço de Neurologia, Hospital de \\ Clínicas de Porto Alegre (HCPA). \\ Porto Alegre, RS, Brasil. \\ 3 Grupo de Pós-graduação em Ciências \\ Médicas, Universidade Federal do \\ Rio Grande do Sul (UFRGS). \\ Porto Alegre, RS, Brasil. \\ 4 Serviço de Fisioterapia, Hospital de \\ Clínicas de Porto Alegre (HCPA). \\ Porto Alegre, RS, Brasil. \\ Corresponding author: \\ Bruna Maciel Catarino \\ bruna.catarino@hotmail.com \\ Escola de Educação Física, Fisioterapia \\ e Dança (Esefid), Universidade Federal \\ do Rio Grande do Sul (UFRGS) \\ Rua Felizardo, 750. \\ 90690-200, Porto Alegre, RS, Brasil.
}


Depression affects up to $50 \%$ of people with MS and is regarded as a common alteration related to vegetative changes and functional disability ${ }^{4}$. Lower Urinary Tract Symptoms (LUTS) are also highly incident in this public; they affect most patients who have had MS for longer periods ${ }^{5}$ and may manifest in several ways: detrusor overactivity (drowsiness, increased voiding frequency and urge incontinence), bladder sphincter dyssynergia, and detrusor insufficiency (associated with residual urine, incomplete emptying, hesitation, and interrupted flow).

Although these disorders are highly prevalent and seem to significantly affect the lives of people with MS, they are often underdiagnosed as suggested by Akkoç et al. ${ }^{6}$, making it difficult to understand the connection between these symptoms and their associated factors.

\section{OBJECTIVE}

The objective of this study was to identify the main urinary symptoms and to correlate them with Quality of Life and with depressive symptoms in women with MS treated at the Hospital de Clínicas of Porto Alegre (HCPA), Brazil.

\section{METHODS}

This is an observational study with a descriptive and correlational design. This study was approved by the Research Ethics Committee of the Hospital de Clínicas of Porto Alegre (CAAE number: 52443615300005327). The fieldwork was carried out from March to September 2016.

\section{Population and sample}

Patients undergoing follow-up treatment at the Multiple Sclerosis outpatient clinic of the Hospital de Clínicas of Porto Alegre participated in the study. Participants were invited while they were in the waiting room for a previously scheduled medical appointment. Thus, the study sample was nonprobabilistic by convenience. The inclusion criteria were: women aged 18 years and over, diagnosed with Relapsing-Remitting MS, and with LUTS (such as frequent urination, nocturia, urinary urgency and/or urinary incontinence). Exclusion criteria were: clinical diagnosis of urinary tract infection, pregnancy or puerperal state, comprising a period of up to 6 months.

\section{Procedures for collection and analysis}

All patients were previously informed about the collection procedures and the purposes of the investigation. When they agreed to participate in the research, they signed an Informed Consent Form.
Collection procedures occurred on the same day. They were performed by the same assessor and occurred in 4 stages:

1) Anamnesis file with information on urinary symptoms (nocturia, urgency, voiding frequency and urinary retention), obstetric history, classification on the Kurtzke Expanded Disability Status Scale (EDSS) ${ }^{7}$ to assess MS, and records of the medications used;

2) Beck Depression Inventory-2 (BDI-II), a self-rated scale containing 21 items, which assesses depression signs and symptoms. The total score ranges from 0 to 63 points; from 0 to 13 points, minimal depression/ no depression; 14 to 19 points, mild depression; 20 to 28 points, moderate depression; and 29 to 63 points, severe depression ${ }^{8}$.

3) Incontinence Impact Questionnaire-7 (IIQ-7-BR), the Urogenital Distress Inventory-6 (UDI-6-BR). Both questionnaires are self-administered instruments, composed of 7 and 6 questions, respectively, with the purpose of measuring the impact of UI on the quality of life of people with MS. IIQ-7-BR has a maximum score of 21 points; the closer to 21, the greater the impact of UI on daily living activities and physical activities. UDI-6-BR is composed of three subscales: the first assessed symptoms of urinary stress (urgency, frequency and pain) (questions 1 and 2); the second assesses stress symptoms (questions 3 and 4); and the third, obstruction/ residue or discomfort in the region (questions 5 and 6 ). It has a maximum score of 18 points; the closer the score is to 18 , the more severe the symptoms ${ }^{9}$. Neither the IIQ-7-BR questionnaire nor the UDI-6-BR has a previously established cut-off point.

4) Multiple Sclerosis Quality of Life Questionnaire (MSQOL-54). It has recently been translated into Portuguese and validated. It is a self-administered instrument with 35 generic questions, 17 specific ones, and 2 separate questions: one about change in health status and the other about sexual satisfaction. The scale items are grouped into 12 subscales that result in two major components: physical and mental health. The scores of the subscales vary from 0 to 100 , and higher values indicate a better quality of life ${ }^{10}$.

The anamnesis form was applied by a professional and the questionnaires were self-administered.

\section{Statistical Analysis}

The sample size was calculated by the Statistical Analysis System (SAS) software, version 9.4, aiming to estimate and test a linear association between the quantitative variables of the Quality of Life Score (MSQOL-54), depressive symptoms (BDI-II) and urinary symptoms (IIQ-7-BR and UDI-6-BR). With a "p" value of $\leq 0.05$, power of $90 \%$ and minimum correlation of 0.6 , a minimum sample size of 39 participants was defined.

The Shapiro-Wilk test was performed to verify the normality of the data. The variables were described by average and standard deviation for continuous quantitative variables with symmetric distribution, 
and median and interquartile range for continuous variables, with asymmetric distribution. Categorical variables were described by absolute frequency and relative frequency. In the inferential analysis, the correlation of the variables was carried out through the Spearman's rank correlation test. All analyses considered a significance level of $5 \%(p=\leq 0.05)$ and were performed by the Statistical Package for Social Sciences (SPSS), version 21.0.

\section{RESULTS}

On average 200 patients were being treated at the outpatient clinic and according to the consultation schedule, the patients that fit the selection criteria were invited until the sample size was reached. A total of 41 women participated in the study, the majority overweight according to the World Health Organization. The average EDSS score was 4.1, classifying the patients as having moderate disabilities caused by MS, according to the classification used by Ferreira et al. ${ }^{11}$ and most of the sample (58.5\%) had the disease for 10 years or more (Table 1 ).
According to the classification of the Beck Depression Inventory-2 (BDI - II), $73.2 \%$ of the participants presented symptoms indicative of depression. Regarding quality of life, the median of the physical component was apparently lower ( $\mathrm{md}=45$ ) when compared to the mental component ( $\mathrm{md}=50$ ), indicating that the physical aspects - such as independence, autonomy, pain, sexual function, energy, fatigue and physical limitations - resulted in lower scores, which may refer to a worse quality of life regarding this aspect. (Table 1).

The most common urinary symptom was urinary urgency, reported by $78 \%$ of the sample, followed by difficulty in emptying the bladder $(70 \%)$. Other characteristics about urinary symptoms are show in Table 2.

A moderate and significant negative correlation $(r=-0.561$ and $p \leq 0.001)$ was found between depression and the physical component of quality of life, and a strong and negative significant correlation $(r=-0.729$ and $p \leq 0.001$ ) was found between depression and the mental component of quality of life, which demonstrates that the more severe the symptoms indicative of depression, the worse the quality of life (Figure 1).

Table 1: Characteristics of the sample.

\begin{tabular}{|c|c|}
\hline Variables & $\mathrm{n}=41$ \\
\hline Age (years) - average $\pm S D$ & $50.1 \pm 9.45$ \\
\hline $\mathrm{BMI}$ - average \pm SD & $26.93 \pm 4.72$ \\
\hline Time since diagnosis (months) - md (P25-P75) & $132(51-192)$ \\
\hline Menopause - n (\%) & $20(49.0)$ \\
\hline \multicolumn{2}{|l|}{ Type of birth - n (\%) } \\
\hline Vaginal delivery & $38(57.6)$ \\
\hline Cesarean delivery & $28(42.4)$ \\
\hline Episiotomy - n (\%) & $10(24.3)$ \\
\hline Multiparous - n (\%) & $22(53.6)$ \\
\hline Nulliparous - n (\%) & $15(36.5)$ \\
\hline Primiparous - n (\%) & $8(19.5)$ \\
\hline Use of pads - n (\%) & $22(53.6)$ \\
\hline \multicolumn{2}{|l|}{ EDSS classification - n (\%) } \\
\hline Mild $(0-3.5)$ & $17(41.5)$ \\
\hline Moderate $(4-6.5)$ & $19(46.3)$ \\
\hline Severe $(7+)$ & $5(12.2)$ \\
\hline \multicolumn{2}{|l|}{ MSQOL - md (P25- P75) } \\
\hline Physical component & $45(37-52)$ \\
\hline Mental component & $50(34.5-57)$ \\
\hline BDI-II- md (P25- P75) & $19(12-26)$ \\
\hline \multicolumn{2}{|l|}{ Classification of depression - n (\%) } \\
\hline No depression & $11(26.8)$ \\
\hline Mild depression & $12(29.3)$ \\
\hline Moderate depression & $11(26.8)$ \\
\hline Severe depression & $7(17.1)$ \\
\hline
\end{tabular}

SD = Standard Deviation; EDSS = Kurtzke Expanded Disability Status Scale; MS = Multiple Sclerosis; $\mathrm{md}=$ median; P25 = Percentile 25; P75 = Percentile 75; MSQOL-BR = Multiple Sclerosis Quality of Life Questionnaire - Portuguese; BDI-II = Beck Depression Inventory-2. 
A strong correlation was found between UDI-6-BR and IIQ-7-BR $(r=0.719$ and $p \leq 0.001)$, indicating that the greater the severity of urinary symptoms, the greater their impact. A weak negative correlation was found between the severity of MS according to EDSS and the urinary symptoms, possibly showing that more severe urinary symptoms manifested in participants with lower disability. Regarding the impact of urinary symptoms, a weak negative correlation was found only with the physical component of quality of life. There was no significant correlation between the other analyzed variables (Table 3).
Table 2: Characteristics of the lower urinary tract symptoms.

\begin{tabular}{ll}
\hline \multicolumn{1}{c}{ Variables } & $\mathbf{n}=\mathbf{4 1}$ \\
\hline Urinary symptoms & $32(78.0)$ \\
Urinary urgency $-\mathrm{n}(\%)$ & $29(70.7)$ \\
Urine retention $-\mathrm{n}(\%)$ & $24(58.5)$ \\
Frequent urination - $\mathrm{n}(\%)$ & $19(46.3)$ \\
Nocturia $-\mathrm{n}(\%)$ & $15(36.5)$ \\
Mixed urinary incontinence - n (\%) & $8(2-11)$ \\
IIQ-7 - md (P25-P75) & $7(4-10)$ \\
UDI- 6 - md (P25 - P75)
\end{tabular}

IIQ-7 = Incontinence Impact Questionnaire-7; UDI-6 = Urogenital Distress Inventory-6; $\mathrm{md}=$ median; $\mathrm{P} 25=$ Percentile 25; P75 = Percentile 75 .

Table 3: Association between the variables through Spearman's rank correlation coefficient.

\begin{tabular}{lcc}
\hline \multicolumn{1}{c}{ Association } & Correlation & poefficient \\
IIQ7 x UDI6 & $0.719^{*}$ & $<0.001$ \\
EDSS x UDI6 & $-0.288^{*}$ & 0.036 \\
IIQ7 x MSQOL - Physical Component & $-0.303^{*}$ & 0.029 \\
IIQ7 x MSQOL - Mental Component & -0.236 & 0.071 \\
UDI6 x MSQOL - Physical Component & -0.138 & 0.198 \\
UDI6 x MSQOL - Mental Component & -0.123 & 0.225 \\
EDSS x IIQ7 & -0.056 & 0.366 \\
EDSS x BDI II & 0.121 & 0.229 \\
EDSS x MSQOL - Physical Component & -0.175 & 0.140 \\
EDSS x MSQOL - Mental Component & 0.136 & 0.202 \\
Time since diagnosis of MS x UDI6 & -0.074 & 0.325 \\
Time since diagnosis of MS x IIQ7 & -0.032 & 0.423 \\
\hline
\end{tabular}

IIQ-7 = Incontinence Impact Questionnaire-7; UDI-6 = Urogenital Distress Inventory-6; EDSS = Kurtzke Expanded Disability Status Scale; Multiple Sclerosis Quality of Life Questionnaire - Portuguese; BDI-II = Beck Depressionventory-2; MS = Multiple Sclerosis

MSOOL - MENTAL COMPONENT ALCOMPONENT

A)

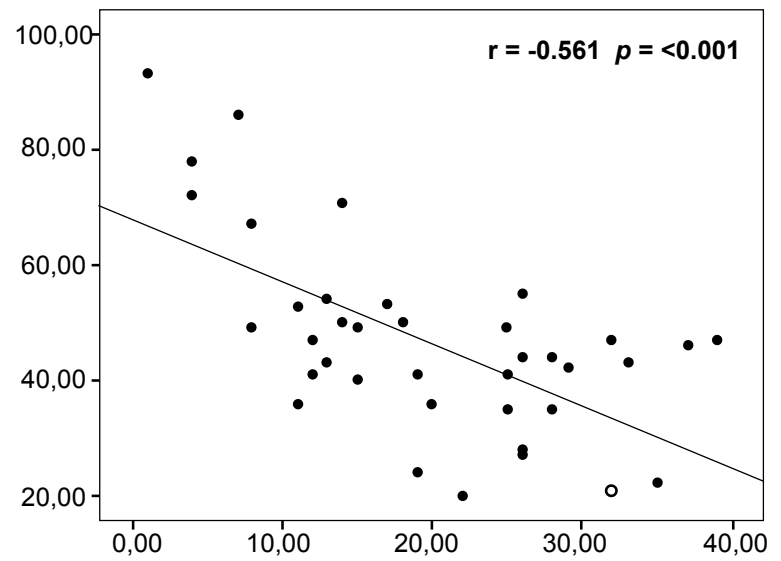

B)

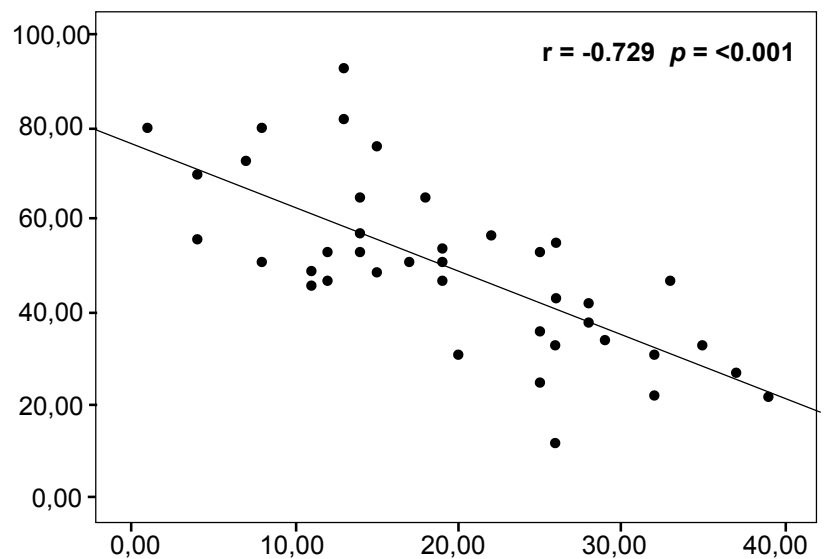

Figure 1: Association between the depression score BDI with the physical $(A)$ and mental $(B)$ components of the MSQOL score.

Regarding the drugs used to control MS, $48.7 \%$ of the patients used glatiramer acetate (Copaxone $\left.{ }^{\circledR}\right), 22 \%$ used Fingolimod (Gilenya ${ }^{\circledR}$ ), and $17 \%$ used Interferon beta-1a $\left(\right.$ Avonex $\left.^{\circledR}\right)$. As to other drugs used by the 
patients, $61 \%$ of the sample used antidepressants, the most common ones including Amitriptyline $(22 \%)$, Fluoxetine $(22 \%)$, and Sertraline $(9.7 \%)$. Regarding diuretic medication, $12.1 \%$ used Hydrochlorothiazide, and, to control urinary symptoms, only $9.7 \%$ used Oxybutynin.

None of the women assessed received treatment for their LUTS previously; after evaluation and identification of the symptoms, all patients received guidance and treatment referral.

\section{DISCUSSION}

The present study aimed to identify the most common urinary symptoms and their impact as well as and depressive symptoms on the quality of life of women with MS undergoing follow-up care at HCPA. The average age of the 41 participants was 50.1 years; the average time since diagnosis of MS was 132 months; the most common urinary symptom was difficulty in emptying the bladder, and no correlation was found between severity and impact of urinary symptoms with quality of life. Depression correlated with poorer quality of life in both the physical and mental components. In the sample studied, vaginal deliveries were more prevalent; $48 \%$ of the sample was postmenopausal, $55 \%$ were multiparous and of the 21 women who underwent a vaginal delivery, $71.4 \%$ had an episiotomy.

As to the age of the participants, time since diagnosis of MS and classification by EDSS, the findings of this study relate to the results of another study that assessed a greater number of participants ${ }^{12}$. The study by Akkoç et al. ${ }^{6}$ found that urinary urgency was the most prevalent symptom (62\%), followed by frequent urination $(50.4 \%)$, Urge Urinary Incontinence (UUI) $(44.7 \%)$, and nocturia (33\%). In this study, urinary urgency was also the most prevalent symptom (78\%), but, different from the findings by Akkoç et al. ${ }^{6}$ difficulty in emptying the bladder was the second most frequently reported urinary symptom, almost as prevalent as urinary urgency $(70.7 \%)$.

In this study, Mixed Urinary Incontinence (MUI) was slightly more prevalent. This finding corroborates the results of previous studies ${ }^{5,13}$ and may be related to the fact that MS patients may present with both detrusor muscle abnormality (urgency symptoms and vesical hyperactivity) and sphincter disorders (stress-related symptoms) $)^{12,14}$. In addition, the studied sample seems to be susceptible to risk factors related to MUI, such as vaginal delivery, more than one pregnancy, and menopause, which may also explain the prevalence of $\mathrm{MUI}$ in this sample and corroborates findings from other studies in which MUI was also more prevalent in samples susceptible to these risk factors ${ }^{15}$.

In this study, it was not possible to establish a relevant correlation between the impact (IIQ-7-BR) and severity (UDI-6-BR) of urinary symptoms with the quality of life of participants. In both questionnaires, the medians of the scores were low, demonstrating that the urinary symptoms presented were not serious and, therefore, did not have a great impact on the quality of life, that is, the urinary symptoms presented did not cause the participants to stop participating in social activities outside of home, meeting with family and friends, performing self-care, hygiene and domestic life activities, for example. One important factor that may be related to the fact that urinary symptoms are not severe in the sample studied is that none of the assessed women was going through the outbreak of MS. It is known that sphincter syndrome symptoms are related to the evolutionary symptoms of the disease, that is, the outbreaks aggravates, or pre-disposes, the appearance of these symptoms ${ }^{16}$. In addition, the urinary symptoms may have been influenced by the drugs used in treatment, such as Amitriptyline, used by $22 \%$ of the sample. This substance has an anticholinergic action, decreasing bladder contractility and minimizing urinary symptoms ${ }^{17}$.

Still, one hypothesis regarding the limitations of the study is the subjectivity of the evaluation of the symptoms through self-administered questionnaires, since the patient's mood and other difficult-to-control variables can interfere in the responses and come up with data that are not so compatible with the severity or reality of the symptoms.

Previous studies ${ }^{14,5,18}$ suggest that the longer the time since the diagnosis of MS, the greater the frequency and severity of urinary symptoms. However, this study found that there was no correlation between the time since diagnosis of MS, severity and the impact of urinary symptoms. In addition, there was a weak correlation between EDSS and the severity of urinary symptoms, indicating that, in this sample, these symptoms did not develop along with disease progression, suggesting the importance of early follow-up treatment for patients with MS, in order to identify these symptoms and prevent them from getting worse. Mahajan et al. ${ }^{19}$ also used the UDI-6-BR to assess urinary symptoms, and found that $65 \%$ of participants had at least one moderate to severe symptom. These symptoms were also assessed by the Overactive Bladder Questionnaire and showed a correlation with longer diagnosis time, with greater physical disability, and lower quality of life scores, corroborating the idea that more severe urinary symptoms have a greater impact on the lives of people with MS, which was not observed in this study.

There was also no correlation between the time since diagnosis of MS and quality of life, corroborating the findings of Albuquerque et al. ${ }^{20}$, which demonstrated that the duration of the disease was not associated with quality of life when assessed by the MSQOL-54. 
This shows the multidimensionality and subjectivity involved in the evaluation of quality of life, since it is influenced by psychological, social, emotional, and family factors, not only by the presence or evolution of a disease.

As to the association between depression and MS, Marrie et al. ${ }^{21}$ demonstrated, in a systematic review, that the association between depression and psychiatric disorders in people with MS is more common in the age group from 45 to 59 years. Depression, along with anxiety, are the most common psychiatric disorders in MS, and can affect up to $70 \%$ and $36 \%$ of people, respectively. The results found by these authors are in line with this study, since 30 patients $(73 \%)$ presented symptoms possibly related to depression, and our sample had a mean age of around 50 years old.

Studies indicate that the development or aggravation of depression in people with MS is related to the impairment of physical abilities, since this impairment causes increased dependence to perform basic activities and causes patients to need more support from relatives and caregivers, often causing anxiety and emotional stress ${ }^{22}$. In this context, the results of this study showed that depression is directly related to the impairment of physical capacities and the emotional aspect of patients with MS. It can also be assumed that depression in people with MS may be related to the type of support they receive from family, caregivers and health professionals, which is an important environmental factor to be considered in the assessment of MS patients using the $\mathrm{ICF}^{23}$.
There was no correlation between depression, quality of life, and the EDSS score. These results may be related to the fact that EDSS is an outpatient assessment tool that only takes into account disabled bodily functions, not emphasizing issues related with activity and participation ${ }^{24}$ components, which are assessed in the quality of life questionnaire used in this research.

A possible bias in the study is the fact that the sample was not random, but by convenience; however, since the aim of the study was to assess only patients with urinary disorders, the type of sampling did not necessarily interfere with the results. Even though the sample size was reached, the sample of this study is relatively small, and multicenter cohort studies with follow-up are necessary to more accurately determine the relationship between the studied variables. This study identified that the most prevalent urinary symptoms in the studied sample were voiding urgency, difficulty in emptying the bladder, and MUI, but no correlation was found between the severity of these symptoms and the quality of life. Regarding the signs and symptoms of depression, a strong correlation of these symptoms with quality of life was found in both the physical and mental aspects. In this sense, the results of this study emphasize the importance of an adequate and early assessment of these symptoms. However, more studies are necessary to better understand the relationship and impact of these outcomes in the life of patients with MS.

\section{REFERENCES}

1. Sospedra M, Martin R. Immunology of multiple sclerosis. Annu Rev Immunol. 2005;23:683-747

2. Noseworthy $\mathrm{JH}$, Lucchinetti C, Rodriguez M, Weinshenker BG. Multiple Sclerosis. N Engl J Med. 2000;343(13):938-52.

3. Gama Pereira AB, Sampaio Lacativa MC, Costa Pereira FF, Papais Alvarenga RM. Prevalence of multiple sclerosis in Brazil: a systematic review. Mult Scler Relat Disord. 2015;4(6):572-9.

4. Feinstein A. Multiple sclerosis and depression. Mult Scler. 2011;17(11):1276-81.

5. Castel-Lacanal E, Gamé X, Clanet M, De Boissezon X, Brassat D, Rischmann P, Marque P. Assessment of a program to encourage the multidisciplinary management of urinary disorders in multiple sclerosis. Neurourol Urodyn. 2017;36(3):706-9.
6. Akkoç $Y$, Ersöz $M$, Yüceyar $N$, Tunç $H$, Köklü K, Yoldaş TK, et al. Overactive bladder symptoms in patients with multiple sclerosis: frequency, severity, diagnosis and treatment. J Spinal Cord Med. 2016;39(2):229-33.

7. Kurtzke, JF. Rating neurologic impairment in multiple sclerosis: an expanded disability status scale (EDSS). Neurology. 1983;33:1444-52.

8. Gomes-Oliveira MH, Gorenstein C, Lotufo Neto F, Andrade LH, Wang YP. Validation of the Brazilian Portuguese version of the beck depression inventory-II in a community sample. Rev Bras Psiquiatr. 2012;34(4):389-94.

9. Stievano LP, Olival GS, Silva RAP, Toller VB, Carabetta EG, Cunha ETS, et al. Validation survey of the impact of urinary incontinence (IIQ-7) and inventory of distress urogenital (UDI-6) - the short scales in patients with multiple sclerosis. Arq. Neuro-Psiquiatr. 2015;73(1):46-51.
10. Soares, R. Validação de um instrumento de avaliação da qualidade de vida de pacientes com esclerose múltipla para o português do Brasil [tese]. Porto Alegre: Universidade Federal do Rio Grande do Sul; 2015.

11 Ferreira MLB, Machado MIM, Vilela ML, Guedes MJ, Ataíde L Jr, Santos S, et al. Epidemiologia de 118 casos de esclerose múltipla com seguimento de 15 anos no centro de referência do hospital da restauração de Pernambuco. Arq Neuropsiquiatr. 2004;62:1027-32.

12. Khan F, Pallant JF, Pallant JI, Brand C, Kilpatrick TJ. A randomised controlled trial: outcomes of bladder rehabilitation in persons with multiple sclerosis. $J$ Neurol Neurosurg Psychiatry. 2010;81(9):1033-8.

13. Onal B, Siva A, Buldu I, Demirkesen O, Cetinel $B$. Voiding dysfunction due to multiple sclerosis: a large scale retrospective analysis. Int Braz $\mathrm{J} \mathrm{Urol.}$ 2009;35(3):326-33. 
14. Pavan K, Miguez PB, Marangoni BEM, Tilbery CP, Lianza S. Comportamento da incontinência urinária em pacientes com esclerose múltipla e a sua influência na qualidade de vida. Med Reabil. 2010;29(1):1-5.

15. Knorst MR, Resende TL, Santos TG, Goldim JR. The effect of outpatient physical therapy intervention on pelvic floor muscles in women with urinary incontinence. Braz J Phys Ther. 2013;17(5):442-9.

16. Moreira MA, Eduardo F, Mendes MF, Tilbery CP. Esclerose Múltipla. Arq Neuropsiquiatr. 2000;58(2-B):460-6.

17. Pranikoff K, Constantino G. The use of amitriptyline in patients with urinary frequency and pain. Urology. 1998;51(5A):179-81.
18. Khalaf KM, Coyne KS, Globe DR, Malone DC, Armstrong EP, Patel V, Burks J. The impact of lower urinary tract symptoms on health-related quality of life among patients with multiple sclerosis. Neurourol Urodyn. 2016;35(1):48-54.

19. Mahajan ST, Patel PB, Marrie RA. Under treatment of overactive bladder symptoms in patients with multiple sclerosis: an ancillary analysis of the NARCOMS Patient Registry. J Urol. 2010;183(4):1432-7.

20. Albuquerque C, Geraldo A, Martins R, Ribeiro O. Quality of life of people with multiple sclerosis: clinical and psychosocial determinants. Procedia Soc Behav Sci. 2015;171:359-65.

21. Marrie RA, Reingold S, Cohen J, Stuve O, Trojano M, Sorensen PS. The incidence and prevalence of psychiatric disorders in multiple sclerosis: a systematic review. Mult Scler. 2015;21(3):305-17.

22. Silva EG, Castro PF. Percepção do paciente portador de esclerose múltipla sobre o diagnóstico e tratamento. Mudanças. 2011;19(12):79-88.

23. Coenen M, Cieza A, Freeman J, Khan F, Miller D, Weise A, et al. The development of ICF core sets for multiple sclerosis: results of the international Consensus Conference. J Neurol. 2011;258(8):1477-88.

24. Browne C, Salmon N, Kehoe M. Bladder dysfunction and quality of life for people with multiple sclerosis. Disabil Rehabil. 2015;37(25):2350-8.

Received: Apr 28, 2019 Accepted: Aug 28, 2019 